九州大学学術情報リポジトリ

Kyushu University Institutional Repository

\title{
A Symbolic Representatioin of Field-Forage- Ruminant Relationships using Polar Form on the Complex Plane
}

Shimojo, Masataka

Laboratory of Animal Feed Science, Division of Animal Science, Department of Animal and Marine Bioresoruce Sciences, Faculty of Agriculture, Kyushu University

Ikeda, Kentaro

Research Fellow, Faculty of Agriculture, Kyushu University

Asano, Yoki

Research Fellow, Faculty of Agriculture, Kyushu University

Ishiwaka, Reiko

他

https://doi.org/10.5109/4506

出版情報：九州大学大学院農学研究院紀要. 47 (2)，pp.359-366，2003-02-01. Faculty of Agriculture, Kyushu University

バージョン :

権利関係 : 


\title{
A Symbolic Representation of Field-Forage-Ruminant Relationships using Polar Form on the Complex Plane
}

\author{
Masataka SHIMOJO', Kentaro IKEDA ${ }^{1)}$, Yoki ASANO ${ }^{1)}$, Reiko ISHIWAKA ${ }^{2}$, \\ Tao SHAO ${ }^{3)}$, Hiroyuki SATO'), Manabu TOBISA, Yutaka NAKANO"), \\ Noriko OHBA $^{5)}$, Yasukatsu YANO ${ }^{6}$ and Yasuhisa MASUDA
}

\author{
Laboratory of Animal Feed Science, Division of Animal Science, Department of Animal \\ and Marine Bioresource Sciences, Faculty of Agriculture, Kyushu University, \\ Fukuoka 812-8581, Japan \\ (Received October 31, 2002 and accepted November 7, 2002)
}

This study suggests that a symbolic representation of field-forage-ruminant relationships is given by a series of $\pi / 2$ rotations, on the complex plane, of polar coordinates describing digestible and indigestible dry matter of forages connected with fields.

\section{INTRODUCTION}

In the ruminant production from meadows or pastures, close relationships among fields, forages and ruminants are of great importance to the cycling of nutrient elements (Whitehead, 2000a, b), because this will lead to sustainable systems of ruminant production from forages (Mannetje, 1994; Peel and Lloveras, 1994; Spedding, 1995). Our previous report (Shimojo et al., 2002) suggested an example of analytic description of field-forage-ruminant relationships by introducing a concept of an intersection of forage production and ruminant production, where animals were related to fields through forages eaten and feces excreted. There will be other analytic ways that might be expected to give symbolic descriptions of these relationships. We tried to apply the complex representation to analytic studies of digestibility or indigestibility changes with growth of forages (Shimojo et al., 1998a, b). In some cases of scientific studies, the complex representation of a subject might be helpful to its understanding when described using polar coordinates and subjected to rotations on the complex plane.

The present study was designed to give a symbolic representation of field-forage -ruminant relationships using rotations on the complex plane of polar coordinates

\footnotetext{
1) Laboratory of Animal Feed Science, Division of Animal Science, Department of Animal and Marine Bioresource Sciences, Graduate School of Bioresource and Bioenvironmental Sciences, Kyushu University

2) Research Fellow of the Japan Society for the Promotion of Science

3) Visiting Research Scientist from the People's Republic of China

4). Kyushu University Farm, Fukuoka 811-2307

5) Research Student, School of Agriculture, Kyushu University

6) Technical Specialist, School of Agriculture, Kyushu University

† Corresponding Author (E-mail: mshimojo@agr.kyushu-u.ac.jp)
} 
describing digestible and indigestible dry matter of forages connected with fields.

\section{SOME PROPERTIES OF COMPLEX REPRESENTATION}

\section{Polar coordinates of $Z$ on the complex plane}

The polar coordinates of $Z$ on the complex plane is given by

$$
Z=r(\cos \theta+i \sin \theta),
$$

where $r=$ the distance from 0 to $Z, \boldsymbol{i}$ =imaginary unit, $\theta=$ angle.

\section{Rotations on the complex plane}

The following transformation of 1 will give $\pm(\pi / 2)$ rotations when used as multiplier. Thus,

$$
\begin{aligned}
1 & =\boldsymbol{i} \cdot(-\boldsymbol{i}) \\
& =\left(\cos \frac{\pi}{2}+\boldsymbol{i} \sin \frac{\pi}{2}\right) \cdot\left\{\cos \left(-\frac{\pi}{2}\right)+\boldsymbol{i} \sin \left(-\frac{\pi}{2}\right)\right\} .
\end{aligned}
$$

In (2), $\cos (\pi / 2)+i \sin (\pi / 2)$ gives $\pi / 2$ rotation ( $\pi / 2$ anticlockwise rotation), and $\cos (-\pi / 2)+\boldsymbol{i} \sin (-\pi / 2)$ gives $-\pi / 2$ rotation $(\pi / 2$ clockwise rotation).

The equality (2) is transformed further as follows;

$$
\begin{aligned}
1= & \left(\cos \frac{\pi}{2}+\boldsymbol{i} \sin \frac{\pi}{2}\right) \cdot\left\{\cos \left(-\frac{\pi}{2}\right)+\boldsymbol{i} \sin \left(-\frac{\pi}{2}\right)\right\} \\
= & \left(\cos \frac{\pi}{2}+\boldsymbol{i} \sin \frac{\pi}{2}\right) \cdot\left(\cos \frac{\pi}{2}-\boldsymbol{i} \sin \frac{\pi}{2}\right) \\
= & (\cos \theta+\boldsymbol{i} \sin \theta) \cdot\left\{\cos \left(\frac{\pi}{2}-\theta\right)+\boldsymbol{i} \sin \left(\frac{\pi}{2}-\theta\right)\right\} \\
& \cdot(\cos \theta-\boldsymbol{i} \sin \theta) \cdot\left\{\cos \left(\frac{\pi}{2}-\theta\right)-\boldsymbol{i} \sin \left(\frac{\pi}{2}-\theta\right)\right\} \\
= & (\cos \theta+\boldsymbol{i} \sin \theta) \cdot(\sin \theta+\boldsymbol{i} \cos \theta) \cdot(\cos \theta-\boldsymbol{i} \sin \theta) \cdot(\sin \theta-\boldsymbol{i} \cos \theta) .
\end{aligned}
$$

This shows the following; $\sin \theta+\boldsymbol{i} \cos \theta$ is given by interchanging real and imaginary parts of $\cos \theta+\boldsymbol{i} \sin \theta, \cos \theta-\boldsymbol{i} \sin \theta$ is the conjugate complex of $\cos \theta+\boldsymbol{i} \sin \theta, \sin \theta-$ $\boldsymbol{i} \cos \theta$ is given by interchanging real and imaginary parts of the conjugate complex of $\cos \theta$ $+\boldsymbol{i} \sin \theta$. The equality (3) shows that if the rotation is made at any $\theta[\theta \neq \pm(\pi / 2)]$ first, then there appears the second polar coordinates formed by interchanging real and imaginary parts of the first polar coordinates in order to recover a quarter turn $[ \pm(\pi / 2)]$.

There is also a reflection of (3) in the imaginary axis. Thus,

$$
1=(-\cos \theta+\boldsymbol{i} \sin \theta) \cdot(-\sin \theta+\boldsymbol{i} \cos \theta) \cdot(-\cos \theta-\boldsymbol{i} \sin \theta) \cdot(-\sin \theta-\boldsymbol{i} \cos \theta) .
$$

Combining the right-hand side of (3) and that of (4) gives a series of $\pi / 2$ rotations and that of $-\pi / 2$ rotations, which will be shown in the following two sections. 


\section{A series of $\pi / 2$ rotations}

This is constructed by the four terms that are arranged in the following order; the 1st term of (3), the 2 nd term of (4), the 3rd term of (4) and the 4 th term of (3). Thus,

$$
\begin{aligned}
& (\cos \theta+\boldsymbol{i} \sin \theta) \stackrel{\times \boldsymbol{i}}{\longrightarrow}(-\sin \theta+\boldsymbol{i} \cos \theta) \stackrel{\times \boldsymbol{i}}{\longrightarrow}(-\cos \theta-\boldsymbol{i} \sin \theta) \\
& \stackrel{\times \boldsymbol{i}}{\longrightarrow}(\sin \theta-\boldsymbol{i} \cos \theta) .
\end{aligned}
$$

\section{A series of $-\pi / 2$ rotations}

This is constructed by the four terms arranged in the following order; the 2nd term of (3), the 3rd term of (3), the 4th term of (4) and the 1st term of (4). Thus,

$$
\begin{aligned}
& (\sin \theta+\boldsymbol{i} \cos \theta) \stackrel{\times-\boldsymbol{i}}{\longrightarrow}(\cos \theta-\boldsymbol{i} \sin \theta) \stackrel{\times-\boldsymbol{i}}{\longrightarrow}(-\sin \theta-\boldsymbol{i} \cos \theta) \\
& \stackrel{\times-\boldsymbol{i}}{\longrightarrow}(-\cos \theta+\boldsymbol{i} \sin \theta) .
\end{aligned}
$$

\section{Relationships}

It is shown from (2) (6) that the complex representation of 1 using polar coordinates gives a series of $\pm(\pi / 2)$ rotations, the anticlockwise rotation and the clockwise rotation which are related through interchanging real and imaginary parts. The present study, however, will focus on $\pi / 2$ rotations.

\section{FIELD-FORAGE-RUMINANT RELATIONSHIPS SUGGESTED FROM $\pi / 2$ ROTATIONS OF POLAR COORDINATES}

In the present study, digestible $(D)$ and indigestible $(I)$ dry matter weight of the forage mass $(W)$ connected with fields are described using polar coordinates on the complex plane. A series of $\pi / 2$ rotations in (5) indicates that four polar coordinates are found in the respective four quadrants.

\section{Polar coordinates of $D$ and $I$ on the complex plane}

(A) Description in the 1st quadrant

The polar coordinates $\left(Z_{1}\right)$ of $D$ and $I$ in the 1st quadrant is given by

$$
\begin{aligned}
Z_{1} & =D+\boldsymbol{i} I \\
& =r(\cos \theta+\boldsymbol{i} \sin \theta),
\end{aligned}
$$

where $r=\sqrt{D^{2}+I^{2}}=\sqrt{(D+\boldsymbol{i} I) \cdot(D-\boldsymbol{i} I)}, D+I=W, D=r \cos \theta, I=r \sin \theta, 0<\theta<\pi / 2$.

$D$ and $I$ will not be determined unless the forage is given to ruminants in the digestion experiment, and thus $D$ and $I$ are a kind of symbolic representation. In the present study, $D$ and $I$ are considered to be visible due to a plus sign $(+)$, therefore, the description in the 1st quadrant is regarded as the field with standing forage composed of $D$ and $I$. They have a common unit, for example $D \mathrm{~kg} / \mathrm{a}$ and $I \mathrm{~kg} / \mathrm{a}$.

(B) Description in the 2nd quadrant

The description in the 2 nd quadrant is given by $\pi / 2$ rotation (=being multiplied by $\boldsymbol{i}$ ) 
of $Z_{1}$. Thus,

$$
\begin{aligned}
Z_{1} \cdot \boldsymbol{i} & =r(\cos \theta+\boldsymbol{i} \sin \theta) \cdot \boldsymbol{i} \\
& =r(-\sin \theta+\boldsymbol{i} \cos \theta),
\end{aligned}
$$

where $-r \sin \theta=-I, r \cos \theta=D$.

The rotation from (7) to (8) gives the following two changes. (i) $I$ has been transferred from the imaginary part of (7) to the real part of (8) with a minus sign (-). (ii) $D$ has been transferred from the real part of (7) to the imaginary part of (8) with a plus sign. Thus, $D$ is visible, but $-I$ is considered to be invisible due to a minus sign. Since it is $D$ that has been absorbed from the digestive tract of ruminants, describing in the 2 nd quadrant is associated with the ruminant production from digestible nutrients of forages. This also suggests that absorbing $D \mathrm{~kg} / \mathrm{a}$ from the digestive tract relates ruminants not only to the forage ( $\mathrm{kg}$ ) but also to the field (a), as shown in our previous report (Shimojo et al., 2002).

(C) Description in the 3 rd quadrant

The description in the $3 \mathrm{rd}$ quadrant is given by $\pi / 2$ rotation of $Z_{\mathbf{1}} \cdot \boldsymbol{i}$. Thus,

$$
\begin{aligned}
Z_{1} \cdot \boldsymbol{i}^{2} & =r(-\sin \theta+\boldsymbol{i} \cos \theta) \cdot \boldsymbol{i} \\
& =r(-\cos \theta-\boldsymbol{i} \sin \theta)
\end{aligned}
$$

where $-r \cos \theta=-D,-r \sin \theta=-I$.

The following two changes occur due to the rotation from (8) to (9). (i) $D$ has been transferred from the imaginary part of (8) to the real part of (9) with a minus sign. (ii) $I$ has been transferred from the real part of (8) to the imaginary part of (9) with a minus sign. Since $-D$ and $-I$ are invisible in the field after harvest and consumption by ruminants, the description in the $3 \mathrm{rd}$ quadrant is regarded as the field without standing forage.

(D) Description in the 4 th quadrant

The description in the 4 rd quadrant is given by $\pi / 2$ rotation of $Z_{\mathbf{1}} \cdot \boldsymbol{i}^{2}$. Thus,

$$
\begin{aligned}
Z_{\mathbf{1}} \cdot \boldsymbol{i}^{3} & =r(-\cos \theta-\boldsymbol{i} \sin \theta) \cdot \boldsymbol{i} \\
& =r(\sin \theta-\boldsymbol{i} \cos \theta)
\end{aligned}
$$

where $r \sin \theta=I,-r \cos \theta=-D$.

The rotation from (9) to (10) gives the following two changes. (i) $I$ has been transferred from the imaginary part of (9) to the real part of (10) with a plus sign. (ii) $D$ has been transferred from the real part of (9) to the imaginary part of (10) with a minus sign. $I$ is visible, but $-D$ is invisible. Since it is $I$ that is indigestible and excreted, describing in the 4 th quadrant is associated with the feces excreted from ruminants.

\section{Plotting on the complex plane}

Fig. 1 shows $Z_{1}, Z_{1} \cdot \boldsymbol{i}, Z_{1} \cdot \dot{\boldsymbol{i}}^{2}$ and $Z_{\mathbf{1}} \cdot \boldsymbol{i}^{3}$ plotted on the complex plane, an outline of the ruminant production from forages connected with fields. This seems to form a sort of cycle through a series of $\pi / 2$ rotations, which will be discussed in the next section. 


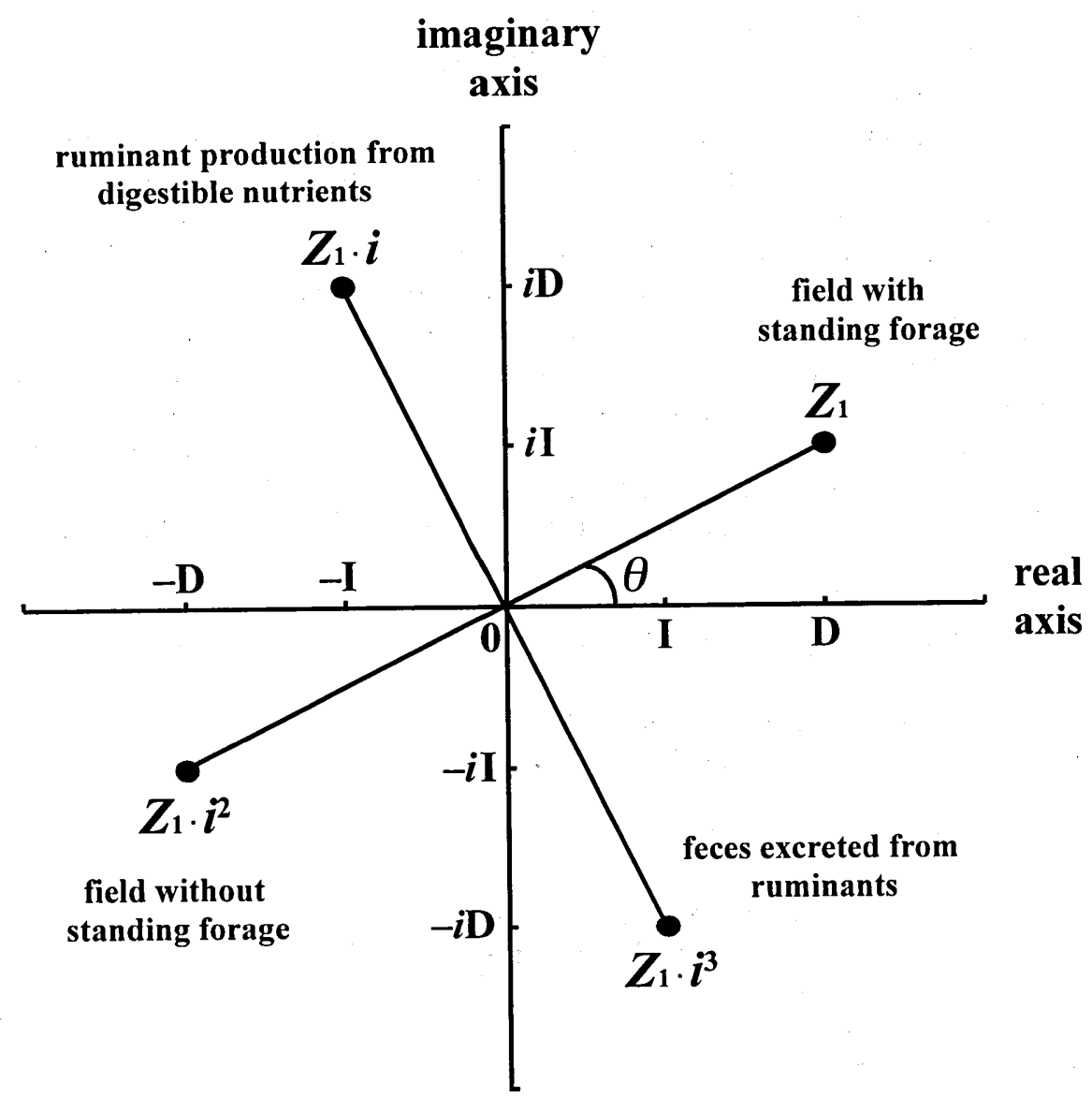

Fig. 1. Plotting of $Z_{1}, Z_{1} \cdot \boldsymbol{i}, Z_{1} \cdot \boldsymbol{i}^{\mathbf{2}}$ and $Z_{1} \cdot \boldsymbol{i}^{\mathbf{3}}$ on the complex plane (D=digestible dry matter of the forage, $I=$ indigestible dry matter of the forage, $i$ =imaginary unit, $\theta=\angle Z_{1} 0 D, Z_{1}$ : field with standing forage, $Z_{1} \cdot i$ : ruminant production from digestible nutrients of the forage, $Z_{1} \cdot \boldsymbol{i}^{2}$ : field without standing forage, $Z_{1} \cdot \boldsymbol{i}^{3}$ : feces excreted from ruminants).

\section{A series of $\pi / 2$ rotations from $Z_{1}$ to $Z_{1} \cdot i^{3}$}

A series of $\pi / 2$ rotations of $Z_{1}$ from the 1 st to the 4 th quadrant is associated with the sum of $Z_{1} \sim Z_{1} \cdot \boldsymbol{i}^{3}$. Thus,

$$
\begin{aligned}
\sum_{\boldsymbol{k}=\mathbf{0}}^{\mathbf{3}} Z_{\mathbf{1}} \cdot \boldsymbol{i}^{\boldsymbol{k}}= & Z_{\mathbf{1}}+Z_{\mathbf{1}} \cdot \boldsymbol{i}+Z_{\mathbf{1}} \cdot \boldsymbol{i}^{2}+Z_{\mathbf{1}} \cdot \boldsymbol{i}^{\mathbf{3}} \\
= & r(\cos \theta+\boldsymbol{i} \sin \theta)+r(-\sin \theta+\boldsymbol{i} \cos \theta)+r(-\cos \theta-\boldsymbol{i} \sin \theta) \\
& +r(\sin \theta-\boldsymbol{i} \cos \theta) \\
= & 0 .
\end{aligned}
$$


This shows that a series of $\pi / 2$ rotations from $Z_{1}$ to $Z_{1} \cdot \boldsymbol{i}^{3}$ forms one cycle of the ruminant production from forages, namely forages through ruminants to feces based on the connection with fields.

If there is a continuation of this cycle, then an additional $\pi / 2$ rotation from $Z_{1} \cdot \boldsymbol{i}^{3}$ to $Z_{1} \cdot \boldsymbol{i}^{4}$ should occur for the onset of the next cycle. This will be achieved by considering the following two things (i) and (ii). (i) $Z_{1} \cdot \boldsymbol{i}^{2}$ shows the field without standing forage, $Z_{1} \cdot \boldsymbol{i}^{3}$ shows the ruminant feces and $Z_{1} \cdot \boldsymbol{i}^{4}$ shows the field with the next standing forage. Therefore, the rotation from $Z_{1} \cdot \boldsymbol{i}^{2}$, through $Z_{1} \cdot \boldsymbol{i}^{3}$, to $Z_{1} \cdot \boldsymbol{i}^{4}$ may be explained as follows; the field without standing forage $\left(Z_{1} \cdot \boldsymbol{i}^{2}\right)$ will become, through receiving the ruminant feces $\left(Z_{1} \cdot \boldsymbol{i}^{3}\right)$, the field with the next standing forage $\left(Z_{1} \cdot \boldsymbol{i}^{4}\right)$. In brief, the next forage production follows the return of ruminant feces to the field after the consumption of the preceding forages by ruminants. (ii) Since the forage productivity changes every harvesting time due to the effects of many environmental and cultivation factors, the following modification $\left(M_{1} \cdot \boldsymbol{i}\right)$ will be given to $Z_{1} \cdot \boldsymbol{i}^{3}$ for obtaining $Z_{2}$ (the next forage production);

$$
M_{1} \cdot \boldsymbol{i}=m_{1}\left(\cos \alpha_{1}+\boldsymbol{i} \sin \alpha_{1}\right) \cdot \boldsymbol{i}
$$

where $m_{1}>0,0<\alpha_{1}<\pi / 2, \boldsymbol{i}=$ imaginary unit.

The product of (10) and (12) gives $Z_{2}$ as follows;

$$
\begin{aligned}
\left(Z_{1} \cdot \boldsymbol{i}^{3}\right) \cdot\left(M_{1} \cdot \boldsymbol{i}\right) & =\left(Z_{1} \cdot M_{1}\right) \cdot \boldsymbol{i}^{4} \\
& =Z_{1} \cdot M_{1} \\
& =\{r(\cos \theta+\boldsymbol{i} \sin \theta)\} \cdot\left\{m_{1}\left(\cos \alpha_{1}+\boldsymbol{i} \sin \alpha_{1}\right)\right\} \\
& =r \cdot m_{1}\left\{\cos \left(\theta+\alpha_{1}\right)+\boldsymbol{i} \sin \left(\theta+\alpha_{1}\right)\right\} \\
& =Z_{2}
\end{aligned}
$$

where $0<\theta+\alpha_{1}<\pi / 2$.

If this cycle repeats $n$ times $(n \geq 2), Z_{n}$ is given by

$$
Z_{n}=Z_{1} \cdot \prod_{k=1}^{n-1} M_{k}
$$

where $Z_{1}=r(\cos \theta+i \sin \theta), M_{k}=m_{k}\left(\cos \alpha_{k}+i \sin \alpha_{k}\right), r>0, m_{k}>0,0<\theta<\pi / 2,0<\alpha_{k}<$ $\pi / 2,0<\theta+\alpha_{1}+\alpha_{2}+\cdots \cdots+\alpha_{k}+\cdots \cdots+\alpha_{n-1}<\pi / 2, \boldsymbol{i}=$ imaginary unit.

Equation (14) is associated with the description of sustainable ruminant production from forages connected with fields when $n$ is as large as possible.

\section{Other properties suggested from $Z_{1}, Z_{1} \cdot i, Z_{1} \cdot i^{2}$ and $Z_{1} \cdot i^{3}$}

$Z_{1}, Z_{1} \cdot \boldsymbol{i}, Z_{1} \cdot \boldsymbol{i}^{2}$ and $Z_{1} \cdot \boldsymbol{i}^{3}$ have so far been regarded as different things one another. Let us consider the following calculations $(C)$ using real and imaginary parts having plus and minus signs (Fig. 1). Thus,

$$
\begin{aligned}
& C \text { for } Z_{1}=\frac{1}{2} \cdot(D) \cdot(I)=\frac{D \cdot I}{2} \\
& C \text { for } Z_{1} \cdot \boldsymbol{i}=\frac{1}{2} \cdot(-I) \cdot(D)=-\frac{D \cdot I}{2} .
\end{aligned}
$$




$$
\begin{aligned}
& C \text { for } Z_{1} \cdot \boldsymbol{i}^{2}=\frac{1}{2} \cdot(-D) \cdot(-I)=\frac{D \cdot I}{2} . \\
& C \text { for } Z_{1} \cdot \boldsymbol{i}^{3}=\frac{1}{2} \cdot(I) \cdot(-D)=-\frac{D \cdot I}{2} .
\end{aligned}
$$

The $C$ for $Z_{1}$ (15) and that for $Z_{1} \cdot \boldsymbol{i}^{2}(17)$ show the same positive value, $(D \cdot I) / 2$. This suggests that both the field with standing forage and the field without standing forage play a role of producer. The calculation form of $(D \cdot I) / 2$ in (15) gives the area of the rectangular triangle in the 1 st quadrant (Fig. 1). In the $3 \mathrm{rd}$ quadrant a minus sign of $-D$ and that of $-I$ are offset by the product of $-D$ and $-I$ in (17), resulting in giving the area of the rectangular triangle.

The same negative value, $-(D \cdot I) / 2$, is found in both $C$ for $Z_{1} \cdot \boldsymbol{i}(16)$ and that for $Z_{\mathbf{1}} \cdot \boldsymbol{i}^{\mathbf{3}}$ (18), because either $D$ or $I$ has a minus sign. This suggests that the ruminant separating the forage mass into nutrients digested and feces excreted plays a role of consumer. Roughly speaking at the risk of making mistakes, $-(D \cdot I) / 2$ might give a negative value to the area of the rectangular triangle. This is actually impossible to accept, but seems to be helpful to understanding different roles in field-forage-ruminant relationships, the consumer 'ruminants' in contrast to the producer 'fields' and 'forages'.

However, modifying the calculations $(15) \sim(18)$ to show the absolute value $(D \cdot I) / 2$ gives no differences among $Z_{1}, Z_{1} \cdot \boldsymbol{i}, Z_{1} \cdot \boldsymbol{i}^{2}$ and $Z_{1} \cdot \boldsymbol{i}^{3}$, the congruity among the four rectangular triangles (Fig. 1). This suggests that forage production (15), ruminant production (16), the field (17) and the feces (18) are both treated equally and related closely in order to form the cycling of nutrient elements in the ruminant agriculture.

In the present study polar coordinates on the complex plane have not been related to Euler's formula,

$$
\exp (\boldsymbol{i} \theta)=\cos \theta+\boldsymbol{i} \sin \theta
$$

This will be taken up in the following report in this issue.

\section{CONCLUSIONS}

It is suggested from the present study that relationships among fields, forages and ruminants are shown symbolically using a series of $\pi / 2$ rotations, on the complex plane, of polar coordinates describing digestible and indigestible dry matter of forages connected with fields.

\section{REFERENCES}

Mannetje, L 't. 1994 Towards sustainable grassland management in The Netherlands. In "Grassland and Society", ed. by L. 't Mannetje and J. Frame, Wageningen Pers, Wageningen, pp. 3-18

Peel, S. and J. Lloveras 1994 New targets for sustainable forage production and utilization. In "Grassland and Society", ed. by L. 't Mannetje and J. Frame, Wageningen Pers, Wageningen, pp. 35-47

Shimojo, M., T. Bungo, Y. Imura, M. Tobisa, N. Koga, S. Tao, M. Yunus, Y. Nakano, I. Goto, M. Furuse and Y. Masuda 1998a Digestibility decrease with forage growth as interpreted using complex plane. Proc. 8th World Conf. Anim. Prod., Seoul, Korea, Vol. II: 514-515

Shimojo, M., T. Bungo, Y. Imura, M. Tobisa, N. Koga, S. Tao, M. Yunus, Y. Nakano, I. Goto, M. Furuse and Y. 
Masuda 1998b Use of complex number in the analysis of increase in dry matter indigestibility with growth of forages. J. Fac. Agr., Kyushu Univ., 43: 137-142

Shimojo, M., K. Ikeda, Y. Asano, R. Ishiwaka, M. Tobisa, T. Shao, N. Ohba, H. Sato, Y. Matsufuji, Y. Yano and Y. Masuda 2002 An intersection of ruminant production and forage production. J. Fac. Agr., Kyushu Univ., 46: 321-329

Spedding, C. R. W. 1995 Sustainability in animal production systems. Anim. Sci., 61: 1-8

Whitehead, D. C. 2000a Introduction. In "Nutrient Elements in Grassland: Soil-Plant-Animal Relationships", CABI Publishing, Oxon, pp. 1-14

Whitehead, D. C. 2000b Nutrient elements in soils. In "Nutrient Elements in Grassland: Soil-Plant-Animal Relationships", CABI Publishing, Oxon, pp. 15-40 